\title{
A prospective study of associations between in utero exposure to gestational diabetes mellitus and metabolomic profiles during late childhood and adolescence
}

\author{
Wei Perng ${ }^{1,2,3}$ (D) Brandy M. Ringham ${ }^{1} \cdot$ Harry A. Smith ${ }^{1,4} \cdot$ Gregory Michelotti $^{5} \cdot$ Katerina M. Kechris $^{4} \cdot$ \\ Dana Dabelea ${ }^{1,2,6}$
}

Received: 26 June 2019 / Accepted: 8 October 2019/Published online: 13 November 2019

(C) Springer-Verlag GmbH Germany, part of Springer Nature 2019

\begin{abstract}
Aims/hypothesis This study aimed to: (1) identify metabolite patterns during late childhood that differ with respect to exposure to maternal gestational diabetes mellitus (GDM); (2) examine the persistence of GDM/metabolite associations 5 years later, during adolescence; and (3) investigate the associations of metabolite patterns with adiposity and metabolic biomarkers from childhood through adolescence.

Methods This study included 592 mother-child pairs with information on GDM exposure $(n=92$ exposed), untargeted metabolomics data at age 6-14 years (T1) and at 12-19 years (T2), and information on adiposity and metabolic risk biomarkers at $\mathrm{T} 1$ and $\mathrm{T} 2$. We first consolidated 767 metabolites at T1 into factors (metabolite patterns) via principal component analysis (PCA) and used multivariable regression to identify factors that differed by GDM exposure, at $\alpha=$ 0.05 . We then examined associations of GDM with individual metabolites within factors of interest at T1 and T2, and investigated associations of GDM-related factors at T1 with adiposity and metabolic risk throughout T1 and T2 using mixed-effects linear regression models.

Results Of the six factors retained from PCA, GDM exposure was associated with greater odds of being in quartile (Q)4 (vs Q1-3) of 'Factor 4' at T1 after accounting for age, sex, race/ethnicity, maternal smoking habits during pregnancy, Tanner stage, physical activity and total energy intake, at $\alpha=0.05$ (OR 1.78 [95\% CI 1.04, 3.04]; $p=0.04$ ). This metabolite pattern comprised phosphatidylcholines, diacylglycerols and phosphatidylethanolamines. GDM was consistently associated with elevations in a subset of individual compounds within this pattern at T1 and T2. While this metabolite pattern was not related to the health outcomes in boys, it corresponded with greater adiposity and a worse metabolic profile among girls throughout the follow-up period. Each 1-unit increment in Factor 4 corresponded with $0.17(0.08,0.25)$ units higher BMI z score, $8.83(5.07,12.59)$ pmol/1 higher fasting insulin, $0.28(0.13,0.43)$ units higher HOMA-IR, and 4.73 (2.15, 7.31) nmol/1 higher leptin.

Conclusions/interpretation Exposure to maternal GDM was nominally associated with a metabolite pattern characterised by elevated serum phospholipids in late childhood and adolescence at $\alpha=0.05$. This metabolite pattern was associated with greater
\end{abstract}

Electronic supplementary material The online version of this article (https://doi.org/10.1007/s00125-019-05036-z) contains peer-reviewed but unedited supplementary material, which is available to authorised users.

Wei Perng

Wei.Perng@CUAnschutz.edu

1 Lifecourse Epidemiology of Adiposity and Diabetes (LEAD) Center, Colorado School of Public Health, University of Colorado Anschutz Medical Campus, Room 208, 12474 E. 19th Ave, Aurora, CO 80045, USA

2 Department of Epidemiology, Colorado School of Public Health, University of Colorado Anschutz Medical Campus, Aurora, CO, USA
3 Department of Nutritional Sciences, School of Public Health, University of Michigan, Ann Arbor, MI, USA

4 Department of Biostatistics and Informatics, Colorado School of Public Health, University of Colorado Anschutz Medical Campus, Aurora, CO, USA

5 Metabolon, Inc., Chapel Hill, NC, USA

6 Department of Pediatrics, School of Medicine, University of Colorado Anschutz Medical Campus, Aurora, CO, USA 


\section{Research in context}

\section{What is already known about this subject?}

- Gestational diabetes mellitus (GDM) is associated with adverse metabolic health in offspring, but little is known of the specific mechanisms and pathways associated with this

- Metabolomics profiling can provide objective information on metabolic pathways linking exposures to health outcomes

- Existing studies of metabolomics of GDM have used maternal serum collected during pregnancy to identify metabolite patterns associated with, or predictive of, development of GDM. They have also investigated metabolite composition in cord blood of offspring exposed to GDM

\section{What is the key question?}

- What are the metabolomics profiles associated with in utero exposure to maternal GDM during childhood and adolescence and how do these correlate with conventional biomarkers of metabolic risk?

\section{What are the new findings?}

- Exposure to maternal GDM is associated with higher circulating phospholipids during childhood (6-14 years of age) and adolescence (12-19 years of age)

- Associations of maternal GDM with metabolites are consistent across 5 years of follow-up, spanning childhood and adolescence

- The phospholipid metabolite pattern is prospectively associated with higher adiposity and worse metabolic profile among girls only

\section{How might this impact on clinical practice in the foreseeable future?}

- A more nuanced understanding of biological pathways linking in utero exposures, like GDM, to adverse health outcomes later in life will inform us of future targets for prevention

adiposity and metabolic risk among female offspring throughout the late childhood-to-adolescence transition. Future studies are warranted to confirm our findings.

Keywords Adiponectin - Adolescence - Gestational diabetes mellitus · Insulin resistance $\cdot$ Leptin · Prospective cohort study Untargeted metabolomics

\author{
Abbreviations \\ 3DPAR 3-Day Physical Activity Recall \\ EPOCH Exploring Perinatal Outcomes among Children \\ GDM Gestational diabetes mellitus \\ ICC Intra-class correlation \\ GPC Glycero-phosphocholine \\ GPE Glycerophosphoethanolamine \\ IQR Interquartile range \\ KPCO Kaiser Permanente of Colorado \\ MET Metabolic equivalent \\ PCA Principal component analysis \\ Q Quartile \\ SAT Subcutaneous adipose tissue \\ SCD Stearoyl- CoA desaturase \\ T1 2006-2009 study period \\ T2 2012-2015 study period (follow-up) \\ VAT Visceral adipose tissue
}

\section{Introduction}

In utero exposure to gestational diabetes mellitus (GDM) is a key risk factor of obesity at birth and beyond [1,2]. In addition to its detrimental effects on offspring adiposity, maternal GDM is also associated with poor metabolic health in offspring later in life, including greater insulin resistance, impaired glucose tolerance or type 2 diabetes, low insulin secretion and alterations in adipokines [3-6]. The effects of maternal GDM on biomarkers of metabolic risk in offspring have been detected as early as 3 years of age [7] and many studies have been able to establish associations of GDM exposure with metabolic biomarkers independent of the child's body composition [6-8], suggesting a unique effect of maternal GDM on in utero 'programming' of the fetus' future metabolic health. 
Metabolomics, the systematic and comprehensive study of low-molecular-weight compounds in biological tissues and fluids, has emerged as a powerful tool to shed light on pathways that link exposures to health outcomes [9]. The majority of published studies on metabolomics in relation to GDM have focused on identifying compounds in maternal fluids (plasma, serum, amniotic fluid, urine) that are associated with and/or predictive of GDM [10-17]. When reviewing the literature, we also identified two studies that compared cord blood metabolite profiles of infants exposed vs those not exposed to GDM $[18,19]$. Both studies leveraged metabolomics data assayed on targeted platforms, which provide information on absolute concentrations of metabolites belonging to a specific but limited set of biochemical pathways hypothesised to be relevant to the research question based on a priori knowledge. Given the scanty knowledge on metabolite profiles associated with GDM exposure in offspring, metabolomics studies employing untargeted, data-driven approaches are needed to comprehensively assess GDM-related metabolic alterations in offspring. Moreover, beyond evaluating associations of GDM with the offspring metabolome during infancy, understanding the influence of maternal GDM on metabolite profiles during childhood and adolescence is important, given that these are vulnerable life stages for the development of excess adiposity [20] and metabolic disease risk [21]. A better understanding of the mechanisms and pathways that link early-life exposures to adverse health outcomes during these time frames will aid in identifying modifiable determinants of these pathways.

In this prospective analysis of over 500 multi-ethnic motherchild dyads, we extend current knowledge surrounding the pathways associated with and consequences of in utero exposure to GDM via three research objectives. First, we sought to identify fasting serum metabolite profiles during late childhood (age 614 years) that differ with respect to in utero exposure to maternal GDM. Next, we assessed the extent to which the relationship between maternal GDM and the metabolites of interest persisted over 5 years of follow-up, into adolescence (age 12-19 years). Finally, in light of evidence in adults that certain metabolite patterns precede worsening of conventional metabolic-disease risk factors [22], we investigated the associations of GDMassociated metabolites with adiposity and conventional biomarkers of metabolic risk from late childhood through adolescence, a vulnerable life stage for the development of excess adiposity [20] and metabolic disease risk [21]. The conceptual model for our research study is depicted in Fig. 1.

\section{Methods}

\section{Study population}

Study participants were from the Exploring Perinatal Outcomes among Children (EPOCH) study, a historical prospective cohort of youth with the original aim of characterising long-term consequences of in utero exposure to maternal diabetes. Details on eligibility and recruitment have been previously published [23]. Between 2006 and 2009 ('T1'), we recruited 604 participants whose mothers were members of the Kaiser Permanente of Colorado (KPCO) health plan. Of them, we excluded children of seven women who had type 1 diabetes, followed by five without sufficient blood volume for untargeted metabolomics profiling for the present study. The analytic sample comprised 592 youth, aged 6-14 years (mean \pm SD age, $10.4 \pm 1.5$ years; interquartile range [IQR], 9.4-11.5 years), with metabolomics data. Of the 592 participants at T1, 403 returned for a followup visit approximately 6 years later, from 2012 to 2015 ('T2'), when participants were $12-19$ years of age (mean \pm SD age, $16.7 \pm 1.2$ years; IQR, 15.9-17.6 years) and had adequate blood volume for metabolomics assays. Figure 2 shows the study population flow. This study was approved by the Colorado Multiple Institutional Review Board (protocol no. 05-0623). All participants provided informed consent.

\section{In utero exposure to maternal GDM}

Exposure to maternal GDM (yes vs no) was defined as a physician's diagnosis of gestational diabetes during the index pregnancy. Diabetes status was ascertained from the KPCO perinatal database, an electronic database that links prenatal and neonatal medical records. All pregnant women who are members of the KPCO Health plan are routinely screened for GDM at 24-28 weeks of gestation using the standard two-step protocol [24]. As previously described [25, 26], GDM was diagnosed when two or more glucose values during the diagnostic $3 \mathrm{~h} 100 \mathrm{~g}$ OGTT met or exceeded the criteria for a positive test [24].

\section{Offspring blood collection}

At T1 T2, trained research assistants collected an $8 \mathrm{~h}$ fasting blood sample from the antecubital vein. All samples were

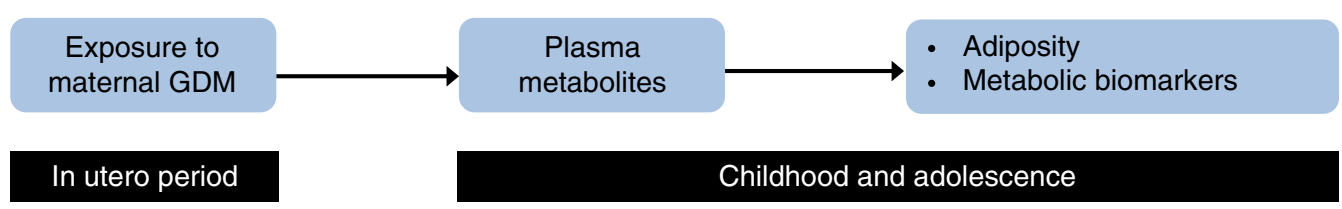

Fig. 1 Conceptual diagram of associations among exposure to maternal GDM, fasting metabolite patterns and health outcomes (adiposity and metabolic biomarkers) throughout childhood and adolescence 


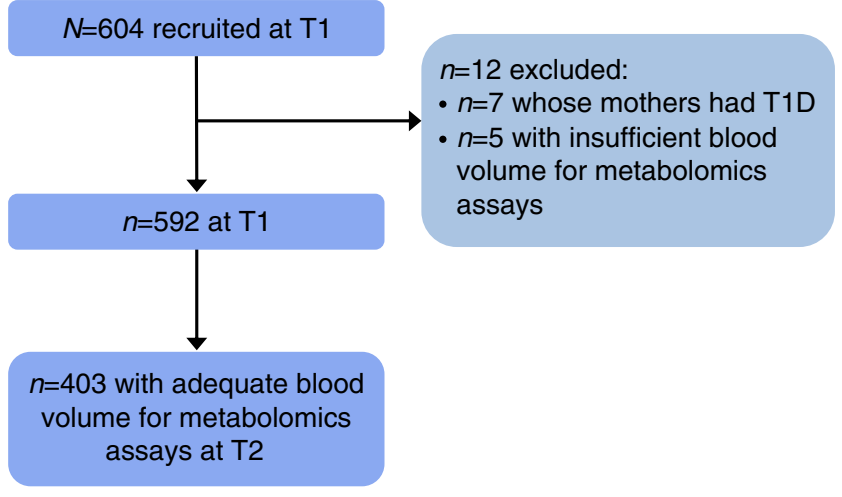

Fig. 2 Study participant flow diagram. T1D, type 1 diabetes

refrigerated immediately, processed within $24 \mathrm{~h}$ and stored at $-80^{\circ} \mathrm{C}$ until the time of analysis. These samples were used for untargeted metabolomics profiling and metabolic biomarker assays.

\section{Untargeted metabolomics profiling}

Metabolon (Morrisville, NC, USA) carried out untargeted metabolomics profiling in fasting plasma collected at $\mathrm{T} 1$ and T2 via a multi-platform mass spectroscopy (MS)-based technique. The procedure identified 1193 unique features at both time points. A key strength of this study is that we sent samples from two research visits for metabolomics profiling at the same time so that technicians were able to balance batches by research visit, thereby enabling comparability of relative metabolite concentrations across the two time points. The electronic supplementary materials (ESM) Methods provides information on sample preparation and laboratory procedures.

We sent participants' serum for metabolomics profiling in two separate batches: the first batch comprised a pilot sample of 100 participants to assess feasibility and the second batch comprised the remaining participants who were not included in the pilot. Prior to formal statistical analysis, we removed metabolites with $\geq 20 \%$ missing values [27] separately for each batch (as is the current recommendation [28]), then imputed the rest of the missing values using the $k$-nearest neighbour algorithm ( $k=10$ neighbours used for imputation) [29]. The first batch of participants had 913 metabolites after removing those with high missingness, and the second batch had 898 metabolites. We then merged the two batches for subsequent data processing. Following the merge, we retained metabolites that were present in both batches (767 compounds) and performed $\log _{10}$-transformation, followed by metabolite normalisation and correction for batch effects (as well as other biological and technical variability) using the 'remove unwanted variation' (RUV) method (the number of factors of unwanted variation estimated from the data $[k]=2$ ), which has proven utility for high-dimensional biological data
[30]. All metabolite processing was performed using $\mathrm{R}$ (Version 3.5.3; Vienna, Austria).

\section{Conventional biomarkers of metabolic risk}

Using fasting blood collected at $\mathrm{T} 1$ and $\mathrm{T} 2$, we assayed fasting glucose enzymatically, and fasting insulin leptin, and adiponectin via radioimmunoassays (Millipore, Darmstadt, Germany). We calculated the HOMA-IR [31]. Fasting insulin, glucose, and estimated insulin resistance are indicators of glycaemic homeostasis in children [31-33] and are associated with future cardiovascular risk [34]. Leptin and adiponectin are adipocyte-derived peptide hormones that regulate weight and metabolism through complementary actions [35]. Increasing evidence suggests that altered adipocytokine levels, specifically elevated leptin, may actually predict subsequent weight gain [36].

\section{Anthropometric and body composition assessment}

At T1 and T2, trained research staff measured height (m) and weight $(\mathrm{kg})$ of participants in light clothing and without shoes. We used these values to calculate BMI $\left(\mathrm{kg} / \mathrm{m}^{2}\right)$ and evaluated this variable in native units, as well as standardised as an age- and sex-specific $z$ score using the WHO growth reference for children aged 5-19 years [37]. As previously described [26], research staff also measured waist circumference $(\mathrm{cm})$ (a correlate of central visceral adiposity $[38,39]$ ) according to the National Health and Nutrition Examination Survey (NHANES) protocol [40]. They also measured subscapular, tricep and suprailiac skinfold thicknesses using Holtain callipers (mm; Crymych, UK) the sum of which ('skinfold sum') was used in the analysis as a proxy for subcutaneous adiposity [39].

A trained technician performed magnetic resonance imaging (MRI) of the abdominal region with a 3 T HDx Imager (General Electric, Waukesha, WI, USA) with the participant in the supine position. A series of spin-lattice relaxation time coronal images were taken to locate the L4/L5 plane. One axial, $10 \mathrm{~mm}$, spin-lattice relaxation time image at the umbilicus or L4/L5 vertebrae was analysed per participant to determine visceral adipose tissue (VAT) and subcutaneous adipose tissue (SAT).

\section{Covariates}

We calculated maternal pre-pregnancy BMI $\left(\mathrm{kg} / \mathrm{m}^{2}\right)$ using clinically recorded pre-pregnancy weight from KPCOderived medical records and measured height at the $\mathrm{T} 1$ visit. At T1, the women filled out a questionnaire regarding treatment received for GDM. Treatment was categorised as diet and/or exercise only $(n=61)$, diet and/or exercise with insulin 
( $n=20)$, and insulin only $(n=5)$. Due to the small sample size for insulin only treatment, we combined this category with diet and/or exercise when using this variable as a covariate in the analysis. At T1, the women also reported on their education level and smoking habits during pregnancy (smoked while pregnant with index child, yes vs no) via a selfadministered questionnaire. In the analysis, we categorised maternal education as a three-level variable: less than high school, high school diploma or equivalent, and higher than high school.

We calculated participants' age at T1 and T2 as the difference between date of each research visit and delivery date. Participants self-reported on their race/ethnicity at $\mathrm{T} 1$ as non-Hispanic white, non-Hispanic black, Hispanic and nonHispanic other. At both research visits, participants reported their pubertal development based on pictorial diagrams of the Tanner stages [41], which had been validated against physician-assessed Tanner staging and puberty-related hormones [42]. We based pubertal status on pubic hair development in boys and breast development in girls. For bivariate analysis, we categorised a child as prepubertal (Tanner stage $=$ 1), pubertal (Tanner stage $=2$ or 3 ), and late/postpubertal (Tanner stage $=4$ or 5 ). In multivariable models, we entered this variable ordinally. We obtained information on the participants' physical activity levels at $\mathrm{T} 1$ and $\mathrm{T} 2$ using the 3-Day Physical Activity Recall (3DPAR) Questionnaire, which captures habitual physical activity based on a 3-day reference period [43]. Using 3DPAR, we derived mean energy expenditure (mean metabolic equivalents [METs]/day over a 3-day period). At both visits, participants completed the Block Kid's Food Frequency Questionnaire (FFQ) [44]. We used these data in conjunction with the United States Department of Agriculture (USDA) Food Composition Database to estimate total energy intake ( $\mathrm{kJ} / \mathrm{day})$.

\section{Data analysis}

Prior to formal analysis, we examined bivariate associations of in utero GDM exposure with maternal, perinatal and child characteristics to identify potential confounders. This step, in conjunction with our a priori knowledge of determinants of metabolic health in youth $[45,46]$, informed the selection of confounders (variables associated with the exposure and a potential determinant of the outcome) and precision covariates (physiological or lifestyle factors that may account for variability in the outcomes of interest) for multivariable analysis. We then carried out the main analysis in three steps, conforming to our study aims.

Step 1: identification of metabolites during late childhood (T1) that differ by GDM exposure To reduce dimensionality of the metabolomics data, we used principal components analysis (PCA) to consolidate the 767 metabolites into latent variables, known as factors, that may be interpreted as metabolite patterns. The procedure generates as many factors as there are original metabolites, so we used visual inspection of the Scree plot for a 'break' (ESM Fig. 1), and standard criterion of eigenvalues $>1$ to determine the number of factors to retain (see ESM Methods for details on PCA).

Next, we examined associations of GDM exposure with the retained factor scores at $\mathrm{T} 1$, which were operationalised two ways: (1) as continuous outcomes (via linear regression); and (2) as a dichotomous outcome (via logistic regression), categorised as quartile (Q)4 vs Q1-3 of the factor score, to allow for potential threshold effects commonly observed in the analyses of potential biological pathways in relation to exposures and health outcomes (e.g., DNA methylation in relation to adiposity [47]). Given the relatively small number of factor scores retained from the PCA, we considered GDM to be associated with a factor score if its $\beta$ value or OR coefficient was significant $(p<0.05)$. In addition, we valued consistency in estimates across multivariable adjustment as this suggests a true relationship between GDM and the factors, as opposed to a spurious association arising from inappropriate covariate adjustment.

We then explored these associations using a series of multivariable models. Figure 3 shows a directed acyclic graph (DAG) of our modelling strategy. Model 1 accounted for key sociodemographic confounders: age, sex, and race/ethnicity. Using this model, we tested for effect modification by sex, for which we observed no evidence ( $p$ interaction $>0.30$ for all), so we included both boys and girls in subsequent models. In Model 2, we accounted for covariates in Model 1 plus mother's smoking habits during pregnancy, an important variable to consider when assessing the impact of the gestational environment on health outcomes in paediatric and adolescent populations [48]. Model 3 further included variables that could affect metabolite composition and thus could account for variability in metabolites (precision covariates): pubertal status, physical activity levels and total energy intake. In Model 4, we further adjusted for GDM treatment. Finally, in Model 5, we included Model 2 covariates plus prepregnancy BMI, which is partly a confounder to the relationship of interest but likely also an overlapping exposure due to the shared intrauterine milieu between maternal overweight/ obesity and GDM [49].

Step 2: investigation of whether associations of GDM with metabolites measured during childhood (T1) persists into adolescence (T2) Based on the findings from 'Step 1', we examined the associations of GDM with key metabolites (i.e., those with factor loading $>|0.50|$ ) within factors of interest at $\mathrm{T} 1$. We also examined associations of GDM with the same metabolites, but measured in plasma collected at $\mathrm{T} 2$. The rationale behind this step was to assess for consistency of associations of GDM with individual metabolites within a 
Fig. 3 Directed acyclic graph (DAG) of confounders and precision covariates in relation to Step 1 of the analysis, which involved investigating the associations of exposure to maternal GDM with metabolite patterns at T1 (age 6-14 years)

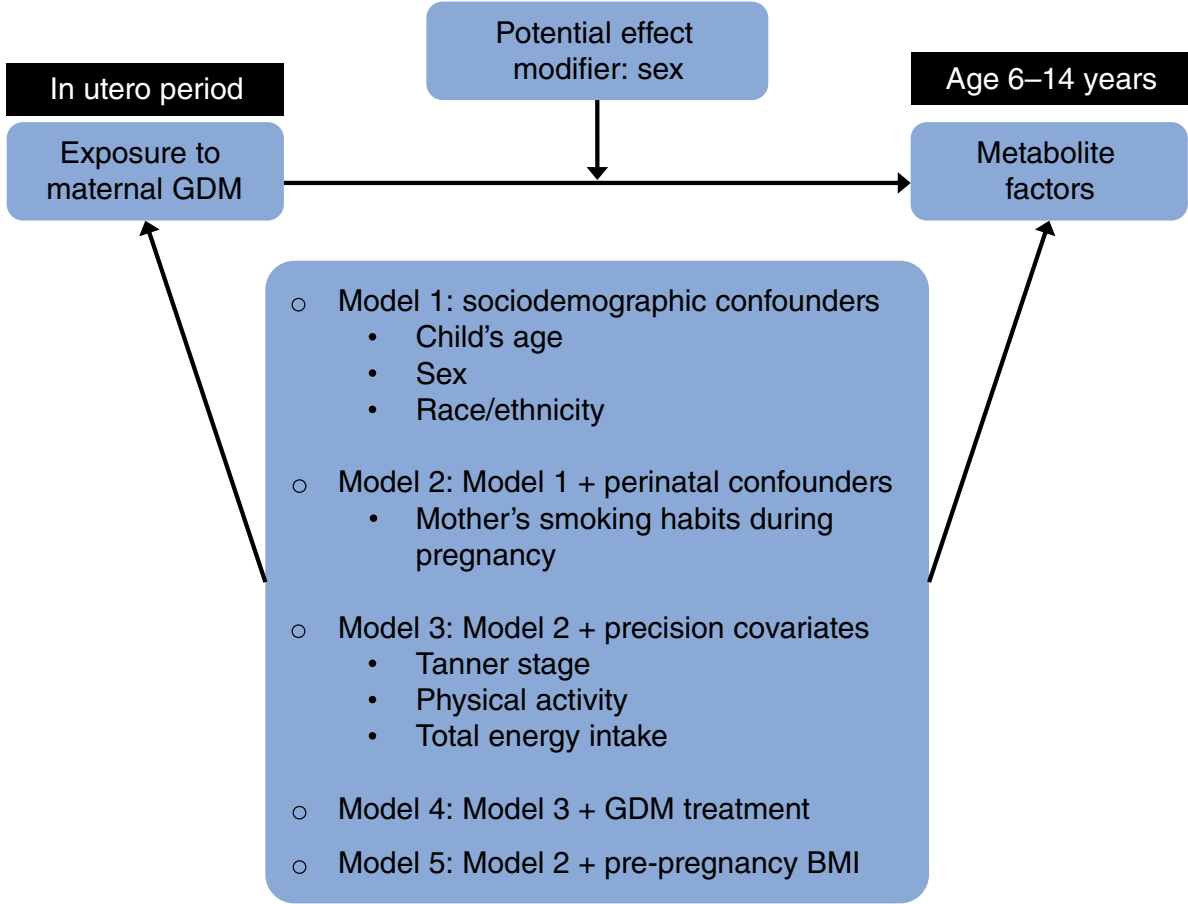

given factor, and to assess whether associations of GDM with metabolites is similar across T1 and T2. For this step, we used multivariable linear regression models that accounted for maternal smoking habits during pregnancy, and child's age, sex and race/ethnicity. We noted all associations with $p<0.05$, but further focused on those that were statistically significant after Bonferroni correction. Findings from this step provided information on inter-individual consistency in associations of GDM exposure with metabolite profiles over time. We also calculated the intra-class correlation (ICC), which assesses intra-individual stability of metabolite concentrations over time.

\section{Step 3: examination of associations of T1 metabolite patterns} with adiposity and metabolic biomarkers throughout T1 and T2 Finally, we examined relationships between the GDMrelated metabolite patterns derived at $\mathrm{T} 1$ with adiposity and metabolic biomarkers from T1 to T2 via mixed-effects linear regression models. The outcomes were the repeated measurements of each adiposity indicator or metabolic biomarker across the two research visits (up to two measurements per outcome per child). The explanatory variables included the metabolite factor score of interest at T1, longitudinal assessments of age, a random effect for the individual study identifier and an unstructured covariance matrix. We chose this approach over evaluating associations of factor scores with health outcomes at T1 and T2 separately, or with change in associations between $\mathrm{T} 1$ and $\mathrm{T} 2$, for model efficiency (i.e., if a participant only had outcome data at $\mathrm{T} 1$, their information still contributed to estimation of standard errors in the model).
Thus, all 592 participants were included in this analysis. Using this model, we tested for an interaction with betweenfactor scores and age and sex and noted several significant $p$ interaction values with respect to sex, even after Bonferroni correction (ESM Table 1), so we ran subsequent multivariable models separately for boys and girls.

In multivariable analysis, we accounted for confounders followed by precision covariates (Fig. 4). Model 1 included child's age, sex and race/ethnicity, Model 2 further accounted for pubertal status and Model 3 included Model 2 covariates plus physical activity and energy intake. When assessing results, we considered all associations that were statistically significant, at $\alpha=0.05$, but also noted significance after Bonferroni correction to account for the number of outcomes assessed by each set of multivariable models; these analyses were carried out separately for boys and girls. In sensitivity analyses, we also evaluated the impact of adjustment for GDM treatment. Doing so did not change the results (data not shown), thus we did not include this variable in the final models.

We carried out all analyses using Statistical Analyses System software (version 9.3; SAS Institute, Cary, NC, USA), unless otherwise stated.

\section{Results}

The mean $( \pm \mathrm{SD})$ age of participants at $\mathrm{T} 1$ was $10.4 \pm 1.5$ years (range, 6.0-13.9 years), and at T2, it was $16.3 \pm 1.2$ years (range, 12.6-19.6 years). Half of the participants were female, 


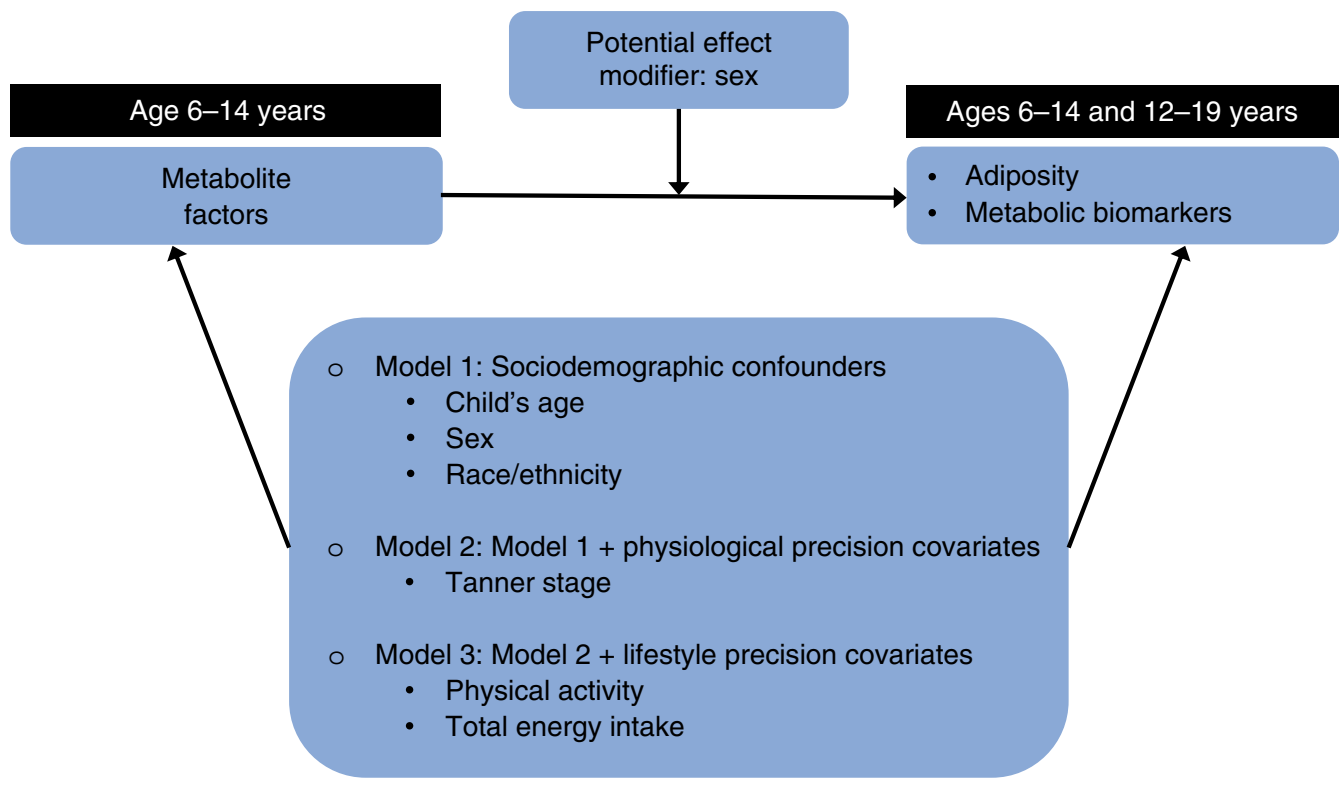

Fig. 4 Directed acyclic graph (DAG) of confounders and precision covariates in relation to Step 3 of the analysis, which involved investigating the associations of metabolite factors at T1 (age 6-14 years) with health outcomes from T1 (age 6-14 years) to T2 (age 12-19 years)

and $48.5 \%(n=287)$ were non-Hispanic white. Approximately $16 \%(n=92)$ of participants were exposed to maternal GDM. Table 1 shows the characteristics of mother-child dyads stratified by GDM exposure status. The GDM-exposed group contained a higher proportion of smokers and non-Hispanic white women vs the unexposed group. With respect to child characteristics, we noted lower fasting glucose $(p=0.006)$, insulin $(p=0.05)$ and HOMA-IR $(p=0.02)$ among GDMexposed vs unexposed participants at T1. The opposite (albeit, non-significant) trends were observed at T2.

We retained six factors (metabolite patterns) that accounted for $50 \%$ of variance in the original metabolomics data at T1 (Fig. 5), which is similar to other studies of untargeted metabolomics data that used PCA for dimension reduction [50]. When we examined the associations of maternal GDM with continuous factor scores using multivariable linear regression, we did not detect any significant associations at $\alpha=0.05$ (i.e., no $p$ values were $<0.05)$. However, when we assessed relationships between GDM and odds of being in Q4 vs Q1-3 of each factor score, we noted a consistent positive association with 'Factor 4'. In comparison with unexposed participants, GDM-exposed youth had 1.64 (95\% CI 0.98, 2.76; $p=0.06)$ greater odds of being in Q4 vs Q1-3 for Factor 4 after adjusting for age, sex and race/ethnicity (Model 1; Table 2 and Fig. 5). Adjustment for maternal smoking (Model 2) did not materially change this estimate. Further adjustment for pubertal status, physical activity, and total energy intake (Model 3) slightly strengthened this association, such that it was statistically significant at $\alpha=0.05$ (OR 1.78 [95\% CI $1.04,3.04] ; p=0.04)$. As expected, this estimate was attenuated to non-significance after accounting for maternal pre- pregnancy BMI (Model 5) (see Fig. 5 and Table 2), suggesting that a large portion of the relationship between maternal GDM and Factor 4 is accounted for by variability in maternal weight status prior to pregnancy. For the above results, none of the estimates were significant after Bonferroni correction.

The metabolite composition of Factor 4 is shown in Table 3; this comprised phosphatidylcholines, lysophospholipids, diacylglycerols and phosphatidylethanolamines. All top-loading metabolites within Factor 4 were positive, indicating that a higher score corresponds with higher serum concentrations of the component metabolites.

Table 4 shows the relationship of maternal GDM with key metabolites within Factor 4 at T1 (i.e., those with factor loading values $>|0.50|$ in the PCA conducted at T1), as well as associations of GDM with the same metabolites at T2. The $\beta$ coefficients for GDM in relation to individual metabolites were similar to the association of GDM with the T1 factor score with respect to the direction of effects. Additionally, GDM was related to the majority of metabolites across $\mathrm{T} 1$ and $\mathrm{T} 2$ in a consistent manner with respect to direction and magnitude of associations. Of the 21 GDMmetabolite associations considered at each time-point, several were significantly different with respect to GDM exposure at both time points using $\alpha=0.05$, but only the estimate for palmitoyl-arachidonoyl-glycerol (16:0/20:4) reached statistical significance after Bonferroni correction (0.09 [95\% CI 0.03, 0.15]; $p=0.002)$. We noted that, although the direction and magnitude of GDM-metabolite associations exhibited preservation of inter-individual rank across the two time points, the ICCs were low-to-modest, with a median (range) of $0.21(0.08-0.38)$. 
Table 1 Bivariate associations of in utero GDM exposure with background characteristics of 592 EPOCH participants

\begin{tabular}{|c|c|c|c|}
\hline Characteristic & GDM-exposed & Unexposed & $p$ \\
\hline$n$ & 92 & 500 & - \\
\hline \multicolumn{4}{|l|}{ Maternal characteristics } \\
\hline Maternal pre-pregnancy BMI $\left(\mathrm{kg} / \mathrm{m}^{2}\right)$ & $27.8 \pm 6.3$ & $25.4 \pm 5.8$ & 0.001 \\
\hline Maternal pre-pregnancy weight status (\%) & & & 0.007 \\
\hline Underweight $\left(\mathrm{BMI}<18.5 \mathrm{~kg} / \mathrm{m}^{2}\right)$ & 1.4 & 3.9 & \\
\hline Normal weight (BMI $18.5-24.9 \mathrm{~kg} / \mathrm{m}^{2}$ ) & 36.5 & 53.8 & \\
\hline Overweight (BMI 25.0-29.9 kg/m²) & 28.4 & 24.0 & \\
\hline Obese $\left(\mathrm{BMI} \geq 30 \mathrm{~kg} / \mathrm{m}^{2}\right)$ & 33.8 & 18.4 & \\
\hline Maternal education level (\%) & & & 0.50 \\
\hline$<$ High school & 5.4 & 3.4 & \\
\hline High school or equivalent & 15.2 & 18.4 & \\
\hline$>$ High school & 79.4 & 78.2 & \\
\hline Smoked during pregnancy $(\%)$ & 16.2 & 7.3 & 0.005 \\
\hline GDM treatment $(\%)$ & 93.5 & & - \\
\hline Diet and/or exercise & 66.3 & - & \\
\hline Diet and/or exercise with insulin, or insulin only & 26.2 & - & \\
\hline None & 6.5 & - & \\
\hline \multicolumn{4}{|l|}{ Child's characteristics } \\
\hline Female $(\%)$ & 46.5 & 50.9 & 0.46 \\
\hline Race/ethnicity (\%) & & & 0.05 \\
\hline Non-Hispanic white & 60.6 & 45.4 & \\
\hline Hispanic & 30.3 & 40.0 & \\
\hline Non-Hispanic black & 5.1 & 8.5 & \\
\hline Non-Hispanic other & 4.0 & 6.1 & \\
\hline \multicolumn{4}{|l|}{ Characteristics at $\mathrm{T} 1$} \\
\hline Age (years) & $9.5 \pm 1.7$ & $10.6 \pm 1.4$ & $<0.0001$ \\
\hline $\mathrm{BMI}\left(\mathrm{kg} / \mathrm{m}^{2}\right)$ & $18.9 \pm 4.7$ & $18.9 \pm 4.5$ & 0.91 \\
\hline BMI $z$ score $^{\mathrm{a}}$ & $0.37 \pm 1.33$ & $0.24 \pm 1.21$ & 0.33 \\
\hline Waist circumference $(\mathrm{cm})$ & $65.4 \pm 12.9$ & $65.5 \pm 12.1$ & 0.98 \\
\hline Skinfold sum $(\mathrm{mm})^{\mathrm{b}}$ & $46.0 \pm 25.5$ & $44.3 \pm 25.4$ & 0.54 \\
\hline SAT fat area $\left(\mathrm{mm}^{2}\right)$ & $125.3 \pm 107.2$ & $119.9 \pm 108.5$ & 0.67 \\
\hline VAT fat area $\left(\mathrm{mm}^{2}\right)$ & $24.1 \pm 18.6$ & $22.1 \pm 16.0$ & 0.30 \\
\hline Fasting glucose (mmol/l) & $4.4 \pm 0.6$ & $4.6 \pm 0.5$ & 0.006 \\
\hline Fasting insulin (pmol/l) & $66.1 \pm 49.8$ & $78.4 \pm 57.2$ & 0.05 \\
\hline HOMA-IR & $1.88 \pm 1.43$ & $2.31 \pm 1.68$ & 0.02 \\
\hline Leptin (nmol/1) & $27.1 \pm 24.6$ & $25.4 \pm 25.8$ & 0.57 \\
\hline Adiponectin $(\mu \mathrm{mol} / \mathrm{l})$ & $77.7 \pm 43.5$ & $78.1 \pm 37.1$ & 0.93 \\
\hline Pubertal stage $(\%)^{\mathrm{c}}$ & & & 0.001 \\
\hline Tanner stage 1 & 71.7 & 51.5 & \\
\hline Tanner stage $2 / 3$ & 22.8 & 42.3 & \\
\hline Tanner stage $4 / 5$ & 5.4 & 6.2 & \\
\hline Energy expenditure (METs/day) ${ }^{\mathrm{d}}$ & $1.85 \pm 0.32$ & $1.90 \pm 0.31$ & 0.13 \\
\hline Total energy intake (kJ/per day) & $72,974 \pm 1916$ & $7523 \pm 2351$ & 0.38 \\
\hline \multicolumn{4}{|l|}{ Characteristics at $\mathrm{T} 2$} \\
\hline Age (years) & $15.9 \pm 1.0$ & $16.8 \pm 1.2$ & $<0.0001$ \\
\hline BMI $\left(\mathrm{kg} / \mathrm{m}^{2}\right)$ & $23.4 \pm 5.0$ & $23.6 \pm 5.7$ & 0.78 \\
\hline BMI $z$ score $^{\mathrm{a}}$ & $0.54 \pm 1.05$ & $0.38 \pm 1.13$ & 0.26 \\
\hline Waist circumference $(\mathrm{cm})$ & $80.9 \pm 11.5$ & $80.8 \pm 13.6$ & 0.92 \\
\hline Skinfold sum $(\mathrm{mm})^{\mathrm{b}}$ & $37.0 \pm 22.2$ & $34.5 \pm 21.3$ & 0.37 \\
\hline
\end{tabular}


Table 1 (continued)

\begin{tabular}{|c|c|c|c|}
\hline Characteristic & GDM-exposed & Unexposed & $p$ \\
\hline SAT fat area $\left(\mathrm{mm}^{2}\right)$ & $200.8 \pm 141.0$ & $200.7 \pm 153.5$ & 0.99 \\
\hline VAT fat area $\left(\mathrm{mm}^{2}\right)$ & $32.9 \pm 19.2$ & $32.8 \pm 22.4$ & 0.98 \\
\hline Fasting glucose $(\mathrm{mmol} / \mathrm{l})$ & $5.2 \pm 2.2$ & $5.0 \pm 0.9$ & 0.16 \\
\hline Fasting insulin (pmol/l) & $124.8 \pm 70.9$ & $114.7 \pm 80.1$ & 0.33 \\
\hline HOMA-IR & $4.31 \pm 3.76$ & $3.85 \pm 4.77$ & 0.44 \\
\hline Leptin (nmol/l) & $40.0 \pm 40.0$ & $41.9 \pm 45.6$ & 0.74 \\
\hline Adiponectin $(\mu \mathrm{mol} / \mathrm{l})$ & $65.7 \pm 32.8$ & $64.1 \pm 34.7$ & 0.72 \\
\hline Pubertal stage $(\%)^{\mathrm{c}}$ & & & 0.85 \\
\hline Tanner stage 1 & 0.0 & 0.0 & \\
\hline Tanner stage $2 / 3$ & 5.6 & 6.2 & \\
\hline Tanner stage $4 / 5$ & 94.4 & 93.8 & \\
\hline Energy expenditure (METs/day) ${ }^{\mathrm{d}}$ & $1.85 \pm 0.27$ & $1.91 \pm 0.32$ & 0.12 \\
\hline Total energy intake (kJ/per day) & $7042 \pm 3209$ & $6916 \pm 3033$ & 0.76 \\
\hline
\end{tabular}

Data are presented as mean $\pm \mathrm{SD}$ or $\%$

${ }^{a}$ Age- and sex-specific $z$ scores according to the WHO Growth Reference for children aged 5-19 years [37]

${ }^{\mathrm{b}}$ Sum of the subscapular, suprailiac and tricep skinfold thicknesses

${ }^{\mathrm{c}}$ Based on breast development for girls and pubic hair development for boys

${ }^{\mathrm{d}}$ Mean metabolic equivalents/day over a 3-day period

Data were analysed using an independent $t$ test for continuous variables and a Pearson's $\chi^{2}$ test for categorical variables

The associations between Factor 4 at $\mathrm{T} 1$ and health outcomes across T1 and T2 within strata of sex are shown in Table 5. In boys, Factor 4 was not associated with adiposity or metabolic risk. In girls, a higher Factor 4 score corresponded with significantly higher adiposity at $\alpha=0.05$ according to all six adiposity indicators (BMI, BMI $z$ score, waist circumference, skinfold sum, SAT fat area and VAT fat area) as well as with higher fasting insulin $(\beta[95 \% \mathrm{CI}]$ per 1-unit factor score: $8.75[4.97,12.53] \mathrm{pmol} / 1 ; p<0.0001)$, higher HOMA-IR $(0.27[0.13,0.40]$ units; $p=0.0002)$ and altered adipokines,

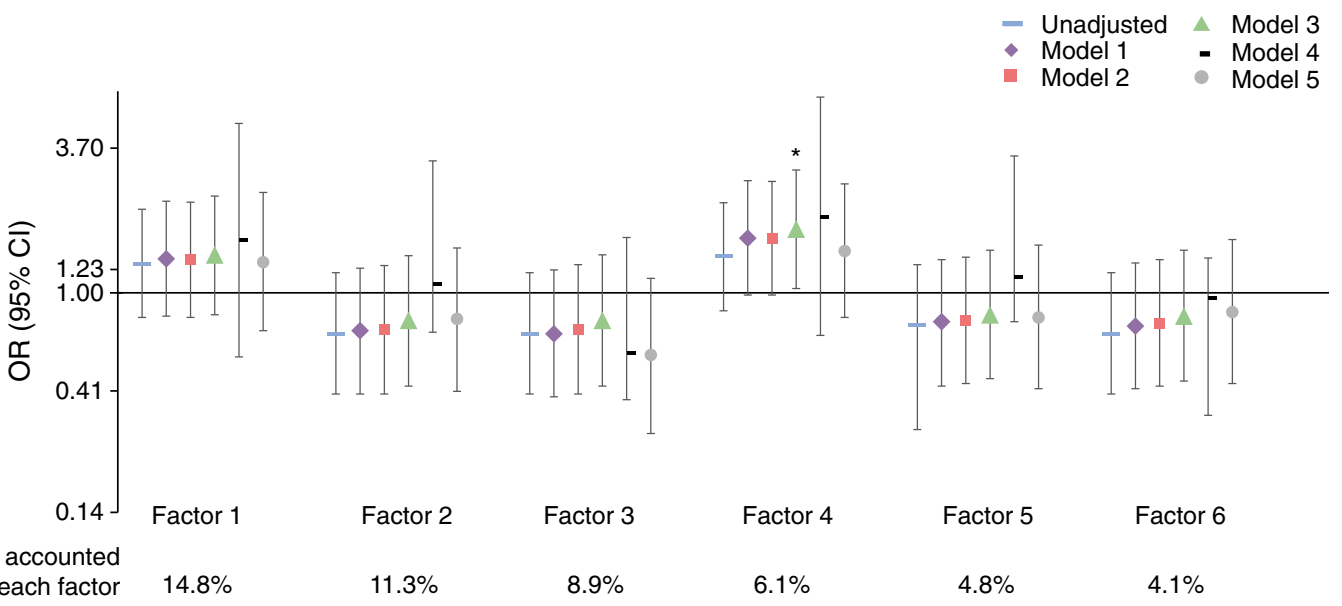

Fig. 5 PCA was used to consolidate 767 metabolites into latent variables ('factors') that may be interpreted as metabolite patterns. Factor scores were selected based on visual inspection of the Scree plot and eigenvalues $>1$. Six factors were retained for use in analysis. Associations of in utero GDM exposure (yes $[n=92]$ vs no $[n=500]$ ) with odds (OR) of being in Q4 vs Q1-3 of each factor score at T1 (age 6-14 years) among the 592 EPOCH participants are shown. The variance accounted for by each factor is indicated on the graph. Model 1: adjusted for child's sex, age

and race/ethnicity; Model 2: Model $1+$ maternal smoking habits during pregnancy (smoking, yes vs no); Model 3: Model $2+$ pubertal status (Tanner stage), physical activity (mean METs/day over a 3-day period) and total energy intake (kJ/day); Model 4: Model 3 + GDM treatment (diet and/or exercise only, diet and/or exercise with insulin or insulin alone, or no treatment); Model 5: Model $2+$ maternal pre-pregnancy BMI $\left(\mathrm{kg} / \mathrm{m}^{2}\right)$. The estimates are plotted on a $\log _{e}$ scale and the horizontal line represents the null (OR 1.00). *Significant at $\alpha=0.05$ 


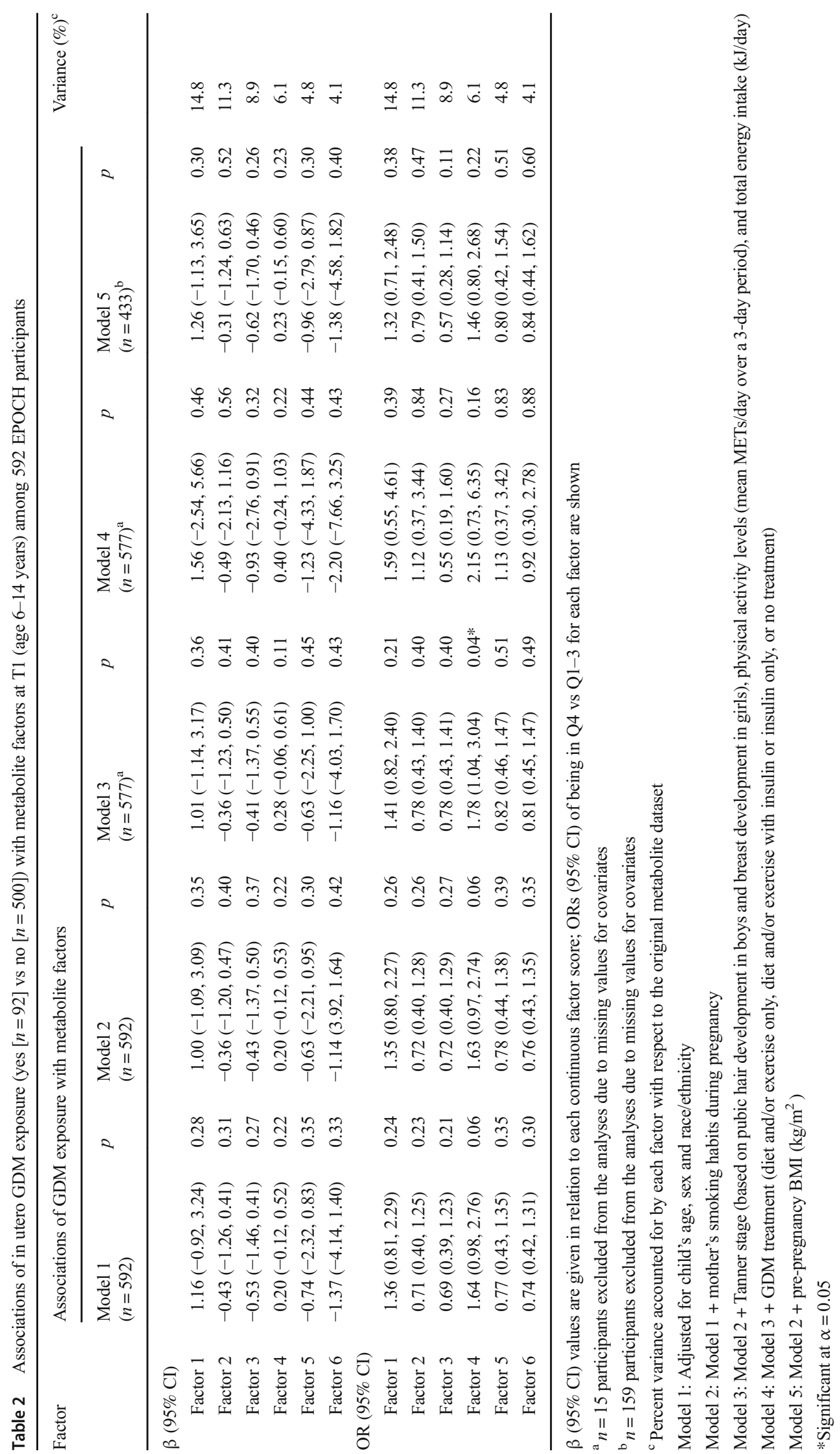


Table 3 Metabolite composition of the GDM-related metabolite pattern ('Factor 4') identified at T1

\begin{tabular}{|c|c|c|c|}
\hline Metabolite & Superpathway & Subpathway & Factor loading \\
\hline Palmitoyl-arachidonoyl-glycerol (16:0/20:4) [tier 2] ${ }^{\mathrm{a}}$ & Lipid & Diacylglycerol & 0.64 \\
\hline 1-Stearoyl-GPE (18:0) & Lipid & Lysophospholipid & 0.64 \\
\hline 1-Palmitoyl-2-palmitoleoyl-GPC (16:0/16:1 $)^{\mathrm{a}}$ & Lipid & Phosphatidylcholine & 0.63 \\
\hline 1-Myristoyl-2-arachidonoyl-GPC (14:0/20:4) ${ }^{\mathrm{a}}$ & Lipid & Phosphatidylcholine & 0.62 \\
\hline Palmitoleoyl-arachidonoyl-glycerol (16:1/20:4) [tier 2] ${ }^{\mathrm{a}}$ & Lipid & Diacylglycerol & 0.62 \\
\hline 1-Palmitoyl-2-arachidonoyl-GPI $(16: 0 / 20: 4)^{\mathrm{a}}$ & Lipid & Phosphatidylinositol & 0.62 \\
\hline 1-Palmitoyl-2-oleoyl-GPE (16:0/18:1) & Lipid & Phosphatidylethanolamine & 0.60 \\
\hline 1-Palmitoyl-GPE (16:0) & Lipid & Lysophospholipid & 0.60 \\
\hline Oleoyl-arachidonoyl-glycerol $(18: 1 / 20: 4)[\text { tier } 1]^{\mathrm{a}}$ & Lipid & Diacylglycerol & 0.60 \\
\hline Oleoyl-arachidonoyl-glycerol (18:1/20:4) [tier 2] ${ }^{\mathrm{a}}$ & Lipid & Diacylglycerol & 0.59 \\
\hline Palmitoyl-oleoyl-glycerol (16:0/18:1) [tier 2] ${ }^{\mathrm{a}}$ & Lipid & Diacylglycerol & 0.59 \\
\hline Palmitoleoyl-linoleoyl-glycerol (16:1/18:2) [tier 1] ${ }^{\mathrm{a}}$ & Lipid & Diacylglycerol & 0.59 \\
\hline Diacylglycerol (16:1/18:2 [tier 2], 16:0/18:3 [tier 1] $)^{\mathrm{a}}$ & Lipid & Diacylglycerol & 0.58 \\
\hline 1-Myristoyl-2-palmitoyl-GPC (14:0/16:0) & Lipid & Phosphatidylcholine & 0.57 \\
\hline Palmitoyl-oleoyl-glycerol (16:0/18:1) [tier 1$]^{\mathrm{a}}$ & Lipid & Diacylglycerol & 0.56 \\
\hline 1-Palmitoleoyl-GPC $(16: 1)^{\mathrm{a}}$ & Lipid & Lysophospholipid & 0.54 \\
\hline 1-Palmitoyl-GPC (16:0) & Lipid & Lysophospholipid & 0.53 \\
\hline 1-Stearoyl-2-oleoyl-GPC (18:0/18:1) & Lipid & Phosphatidylcholine & 0.53 \\
\hline 1-Stearoyl-2-oleoyl-GPE (18:0/18:1) & Lipid & Phosphatidylethanolamine & 0.53 \\
\hline 1-Linolenoyl-GPC $(18: 3)^{\mathrm{a}}$ & Lipid & Lysophospholipid & 0.52 \\
\hline 1-Palmitoyl-GPG $(16: 0)^{\mathrm{a}}$ & Lipid & Lysophospholipid & 0.51 \\
\hline
\end{tabular}

${ }^{a}$ Tier 2 identification in which no commercially available authentic standards could be found, but annotated based on accurate mass, spectral and chromatographic similarity to tier 1-identified compounds

GPG, glycerophosphoglycerol; GPI, glycosylphosphatidylinositol

as indicated by higher leptin $(4.54[1.96,7.11] \mathrm{nmol} / 1 ; p=$ $0.0006)$ and lower adiponectin $(-4.00[-6.76,-1.23]$ $\mu \mathrm{mol} / \mathrm{l} ; p=0.005$ ) (Table 5, Model 1). Further adjustment for pubertal status and lifestyle characteristics in Models 2 and 3 did not greatly change these findings. All abovementioned estimates remained statistically significant after Bonferroni correction $(\alpha<0.05 / 20=0.003)$ except for adiponectin.

\section{Discussion}

\section{Statement of principal findings}

In this study of 592 mother-child dyads, associations of in utero exposure to maternal GDM with offspring metabolite profiles derived from PCA were largely null after correcting for multiple comparisons and following adjustment for maternal pre-pregnancy BMI. However, across several multivariable models that accounted for key perinatal, sociodemographic, biological and lifestyle covariates, we noted that GDM exposure was consistently associated with a fasting serum phospholipid metabolite pattern during late childhood. This metabolite pattern was, in turn, associated with higher adiposity and a worse metabolic risk profile from late childhood through adolescence. We discuss these findings, below.

\section{Associations of maternal GDM with offspring metabolite} profiles at age 6-14 years When assessing offspring metabolite profiles during late childhood, we observed a consistently positive relationship between maternal GDM and a phospholipid metabolite pattern ('Factor 4') that persisted after accounting for maternal smoking habits during pregnancy, and offspring pubertal status, physical activity and energy intake. However, none of the estimates qualified as statistically significant after correcting for multiple testing, and the specific relationship between GDM and Factor 4 was attenuated to non-significance after accounting for maternal prepregnancy BMI. While it is difficult to derive a concrete reason for the former other than limited statistical power, the latter is likely due to the existence of overlapping biological pathways linking maternal overweight/obesity and GDM to offspring metabolism [51] given that maternal glucose is elevated among overweight/obese women, even if they do not qualify as having overt diabetes [49]. Thus, such adjustment is likely to obscure, rather than isolate, the association of maternal glucose levels with offspring outcomes. Additional mechanistic work is required to untangle the unique influence 
Table 4 Associations of in utero GDM exposure with Factor 4 metabolites at T1 $(n=592)$ and T2 $(n=403)$

Factor 4 metabolites

$\mathrm{ICC}^{\mathrm{a}}$

\begin{tabular}{|c|c|c|c|c|c|}
\hline & & \\
\hline & & \multicolumn{2}{|l|}{$\mathrm{T} 1$} & \multicolumn{2}{|l|}{$\mathrm{T} 2$} \\
\hline & & $\beta(95 \% \mathrm{CI})$ & $p$ & $\beta(95 \% \mathrm{CI})$ & $p$ \\
\hline Palmitoyl-arachidonoyl-glycerol (16:0/20:4) [tier 2] & 0.23 & $0.09(0.03,0.15)$ & $0.002 * \dagger$ & $0.07(0.01,0.14)$ & $0.03 *$ \\
\hline 1-Stearoyl-GPE (18:0) & 0.38 & $0.02(-0.01,0.05)$ & 0.16 & $0.01(-0.02,0.04)$ & 0.63 \\
\hline 1-Palmitoyl-2-palmitoleoyl-GPC $(16: 0 / 16: 1)^{\mathrm{b}}$ & 0.21 & $0.00(-0.04,0.04)$ & 0.98 & $0.03(-0.02,0.08)$ & 0.32 \\
\hline 1-Myristoyl-2-arachidonoyl-GPC (14:0/20:4) ${ }^{\mathrm{b}}$ & 0.16 & $0.04(-0.01,0.09)$ & 0.11 & $0.04(-0.02,0.10)$ & 0.17 \\
\hline Palmitoleoyl-arachidonoyl-glycerol (16:1/20:4) [tier 2] & 0.31 & $0.08(0.02,0.15)$ & $0.02 *$ & $0.04(-0.04,0.11)$ & 0.32 \\
\hline 1-Palmitoyl-2-arachidonoyl-GPI (16:0/20:4) ${ }^{\mathrm{b}}$ & 0.22 & $0.05(0.00,0.09)$ & $0.04 *$ & $0.04(-0.02,0.09)$ & 0.17 \\
\hline 1-Palmitoyl-2-oleoyl-GPE (16:0/18:1) & 0.20 & $0.03(-0.02,0.08)$ & 0.24 & $0.03(-0.03,0.10)$ & 0.31 \\
\hline 1-Palmitoyl-GPE (16:0) & 0.38 & $0.00(-0.03,0.04)$ & 0.90 & $0.00(-0.04,0.04)$ & 0.86 \\
\hline Oleoyl-arachidonoyl-glycerol (18:1/20:4) [tier 1$]^{\mathrm{b}}$ & 0.33 & $0.05(0.01,0.09)$ & $0.02 *$ & $0.06(0.02,0.11)$ & $0.009^{*}$ \\
\hline Oleoyl-arachidonoyl-glycerol (18:1/20:4) [tier 2$]^{\mathrm{b}}$ & 0.35 & $0.04(0.00,0.09)$ & 0.07 & $0.05(0.00,0.10)$ & $0.05 *$ \\
\hline Palmitoyl-oleoyl-glycerol (16:0/18:1) [tier 2] ${ }^{\mathrm{b}}$ & 0.17 & $0.07(0.02,0.12)$ & $0.01 *$ & $0.10(0.03,0.17)$ & $0.005^{*}$ \\
\hline Palmitoleoyl-linoleoyl-glycerol (16:1/18:2) [tier 1] ${ }^{\mathrm{b}}$ & 0.28 & $0.08(0.03,0.14)$ & $0.003 *$ & $0.07(0.00,0.14)$ & $0.04 *$ \\
\hline Diacylglycerol (16:1/18:2 [tier 2], 16:0/18:3 [tier 1] $)^{\mathrm{b}}$ & 0.29 & $0.07(0.01,0.12)$ & $0.03 *$ & $0.04(-0.03,0.11)$ & 0.24 \\
\hline 1-Myristoyl-2-palmitoyl-GPC (14:0/16:0) & 0.18 & $-0.02(-0.07,0.03)$ & 0.38 & $0.02(-0.05,0.08)$ & 0.59 \\
\hline Palmitoyl-oleoyl-glycerol (16:0/18:1) [tier 1] ${ }^{\mathrm{b}}$ & 0.16 & $0.07(0.02,0.13)$ & $0.01 *$ & $0.09(0.02,0.16)$ & $0.008 *$ \\
\hline 1-Palmitoleoyl-GPC $(16: 1)^{\mathrm{b}}$ & 0.21 & $0.01(-0.02,0.04)$ & 0.58 & $0.02(-0.01,0.05)$ & 0.17 \\
\hline 1-Palmitoyl-GPC (16:0) & 0.08 & $0.01(-0.01,0.02)$ & 0.32 & $0.00(-0.01,0.02)$ & 0.73 \\
\hline 1-Stearoyl-2-oleoyl-GPC (18:0/18:1) & 0.21 & $0.00(-0.03,0.02)$ & 0.95 & $0.00(-0.03,0.03)$ & 0.85 \\
\hline 1-Stearoyl-2-oleoyl-GPE (18:0/18:1) & 0.18 & $0.04(-0.01,0.09)$ & 0.15 & $0.03(-0.03,0.10)$ & 0.28 \\
\hline 1-Linolenoyl-GPC $(18: 3)^{\mathrm{b}}$ & 0.09 & $0.00(-0.04,0.04)$ & 0.90 & $0.01(-0.04,0.05)$ & 0.73 \\
\hline 1-Palmitoyl-GPG $(16: 0)^{\mathrm{b}}$ & 0.15 & $0.03(-0.01,0.07)$ & 0.16 & $0.00(-0.04,0.05)$ & 0.87 \\
\hline
\end{tabular}

Metabolite concentrations were $\log _{10}$-transformed prior to use in the regression models that generated the estimates displayed in the table. $\beta$ estimates are adjusted for maternal smoking habits during pregnancy, and child's age at research visit, sex and race/ethnicity

${ }^{\mathrm{a}}$ ICC for each metabolite across $\mathrm{T} 1$ and $\mathrm{T} 2$

${ }^{\mathrm{b}}$ Tier 2 identification (denoted by [2]) in which no commercially available authentic standards could be found, but annotated based on accurate mass, spectral and chromatographic similarity to tier 1-identified compounds (denoted by [1])

*Significant at $\alpha=0.05 ;{ }^{\dagger}$ Significant after Bonferroni correction $(\alpha<0.05 / 21=0.002$ at each time point $)$

GPG, glycerophosphoglycerol; GPI, glycosylphosphatidylinositol

of maternal blood glucose levels on offspring health outcomes, independent of weight status entering pregnancy.

The top metabolites in Factor 4 included phospholipids of the palmitoleic acid moiety, including 1-palmitoyl-2palmitoleoyl-glycero-phosphocholine (GPC) (16:0/16:1), palmitoyl-arachidonoyl-glycerol (16:0/20:4), 1-myristoyl-2arachidonoyl-GPC (14:0/20:4), 1-palmitoyl-2-arachidonoylglycosylphosphatidylinositol (GPI) (16:0/20:4), 1-palmitoyl2-oleoyl- glycerophosphoethanolamine (GPE) (16:0/18:1) and 1-palmitoyl-GPE (16:0). This pattern also captured some diacylglycerides, including palmitoyl-linoleoyl-glycerol $(16: 1 / 18: 2)$, which is a common emulsifier used in bakery products, shortening, whipped toppings and other confections [52]. Endogenously, alterations in phospholipid composition could be indicative of either lipolytic or lipogenic activity, both of which have implications for metabolic health, but we are not able to make this distinction in this study. However, we note that palmitate enrichment of fatty acids in this metabolite pattern may be driven by stearoyl-CoA desaturase (SCD), an endoplasmic reticulum enzyme that catalyses the rate-limiting step in monounsaturated fatty acid formation, including palmitoleate from stearoyl-CoA and palmitoyl-CoA [53]. Given that insulin resistance is related to disruptions to the SCD pathway $[54,55]$, it makes sense that this metabolite pattern differed with respect to status of in utero exposure to GDM.

In a recently-published meta-analysis of four cohorts of diverse ancestry, Lowe et al [18] analysed targeted metabolomics data from cord blood of 1600 mother-infant pairs in relation to maternal fasting glucose, glucose at 1-h and 2-h post OGTT, and insulin sensitivity. Key findings included associations of higher 1-h post-OGTT glucose with higher cord blood levels of the ketone body 3-hydroxybutyrate and its carnitine ester, as well as glycerol and 3-hydroxy-decanoyl 
Table 5 Associations of Factor 4 with adiposity and metabolic risk biomarkers from T1 (age 6-14 years) through T2 (age 12-19 years) among 592 EPOCH participants

\begin{tabular}{|c|c|c|c|c|c|c|}
\hline \multirow[t]{2}{*}{ Variable } & \multicolumn{2}{|l|}{ Model 1} & \multicolumn{2}{|l|}{ Model 2} & \multicolumn{2}{|l|}{ Model 3} \\
\hline & $\beta(95 \% \mathrm{CI})$ & $p$ & $\beta(95 \% \mathrm{CI})$ & $p$ & $\beta(95 \% \mathrm{CI})$ & $p$ \\
\hline \multicolumn{7}{|l|}{ Boys $(n=295)$} \\
\hline \multicolumn{7}{|l|}{ Adiposity } \\
\hline BMI $\left(\mathrm{kg} / \mathrm{m}^{2}\right)$ & $0.22(-0.18,0.62)$ & 0.29 & $0.23(-0.17,0.62)$ & 0.27 & $0.21(-0.19,0.62)$ & 0.30 \\
\hline BMI $z$ score $^{\mathrm{a}}$ & $0.09(-0.01,0.18)$ & 0.07 & $0.09(0.00,0.18)$ & 0.06 & $0.09(-0.01,0.18)$ & 0.08 \\
\hline Waist circumference $(\mathrm{cm})$ & $0.42(-0.61,1.44)$ & 0.42 & $0.43(-0.60,1.46)$ & 0.41 & $0.41(-0.64,1.45)$ & 0.45 \\
\hline Skinfold sum $(\mathrm{mm})^{\mathrm{b}}$ & $1.21(-0.85,3.27)$ & 0.25 & $1.22(-0.84,3.29)$ & 0.24 & $1.03(-1.08,3.13)$ & 0.34 \\
\hline SAT fat area $\left(\mathrm{mm}^{2}\right)$ & $4.21(-6.81,15.22)$ & 0.45 & $4.56(-6.38,15.50)$ & 0.41 & $4.28(-6.90,15.45)$ & 0.45 \\
\hline VAT fat area $\left(\mathrm{mm}^{2}\right)$ & $0.87(-0.82,2.57)$ & 0.31 & $0.84(-0.87,2.55)$ & 0.34 & $0.74(-1.01,2.49)$ & 0.40 \\
\hline \multicolumn{7}{|l|}{ Metabolic biomarkers } \\
\hline Fasting glucose $(\mathrm{mmol} / \mathrm{l})$ & $-0.03(-0.06,0.01)$ & 0.11 & $-0.02(-0.06,0.01)$ & 0.13 & $-0.03(-0.06,0.00)$ & 0.09 \\
\hline Fasting insulin (pmol/l) & $2.13(-2.19,6.44)$ & 0.33 & $2.26(-2.00,6.52)$ & 0.30 & $2.09(-2.29,6.46)$ & 0.35 \\
\hline HOMA-IR & $0.03(-0.12,0.18)$ & 0.71 & $0.03(-0.11,0.18)$ & 0.65 & $0.03(-0.12,0.18)$ & 0.73 \\
\hline Leptin (nmol/1) & $1.22(-0.85,3.28)$ & 0.25 & $1.21(-0.85,3.27)$ & 0.25 & $1.06(-1.06,3.19)$ & 0.32 \\
\hline Adiponectin $(\mu \mathrm{mol} / \mathrm{l})$ & $-1.03(-3.79,1.72)$ & 0.46 & $-1.03(-3.79,1.74)$ & 0.46 & $-1.23(-4.06,1.60)$ & 0.39 \\
\hline \multicolumn{7}{|l|}{ Girls $(n=297)$} \\
\hline \multicolumn{7}{|l|}{ Adiposity } \\
\hline BMI $\left(\mathrm{kg} / \mathrm{m}^{2}\right)$ & $0.65(0.31,0.99)$ & $0.0002 * \dagger$ & $0.65(0.32,0.99)$ & $0.0002 * \dagger$ & $0.64(0.31,0.98)$ & $0.0002 * \dagger$ \\
\hline BMI $z$ score $^{\mathrm{a}}$ & $0.17(0.08,0.26)$ & $0.0001 * \dagger$ & $0.17(0.09,0.25)$ & $<0.0001 * \dagger$ & $0.17(0.08,0.25)$ & $0.0001 * \dagger$ \\
\hline Waist circumference $(\mathrm{cm})$ & $1.66(0.86,2.45)$ & $<0.0001 * \dagger$ & $1.67(0.88,2.45)$ & $<0.0001 * \dagger$ & $1.63(0.85,2.41)$ & $<0.0001 * \dot{t}$ \\
\hline Skinfold sum $(\mathrm{mm})^{\mathrm{b}}$ & $4.02(2.33,5.70)$ & $<0.0001 * \dagger$ & $4.02(2.34,5.70)$ & $<0.0001 * \dagger$ & $4.06(2.38,5.75)$ & $<0.0001 * \dagger$ \\
\hline SAT fat area $\left(\mathrm{mm}^{2}\right)$ & $16.22(8.00,24.44)$ & $0.0001 * \dagger$ & $16.23(8.03,24.44)$ & $0.0001 * \dagger$ & $16.27(8.21,24.74)$ & $0.0001 * \dagger$ \\
\hline VAT fat area $\left(\mathrm{mm}^{2}\right)$ & $2.22(0.85,3.58)$ & $0.002 * \dagger$ & $2.21(0.84,3.58)$ & $0.002 * \dagger$ & $2.17(0.78,3.56)$ & $0.002 * \dagger$ \\
\hline \multicolumn{7}{|l|}{ Metabolic biomarkers } \\
\hline Fasting glucose $(\mathrm{mmol} / \mathrm{l})$ & $0.00(-0.04,0.04)$ & 0.99 & $0.00(-0.04,0.04)$ & 0.99 & $0.01(-0.04,0.06)$ & 0.80 \\
\hline Fasting insulin (pmol/l) & $8.75(4.97,12.53)$ & $<0.0001 * \dagger$ & $8.83(5.17,12.49)$ & $<0.0001 * \dagger$ & $8.83(5.07,12.59)$ & $<0.0001^{* \dagger}$ \\
\hline HOMA-IR & $0.27(0.13,0.41)$ & $0.0002 * \dagger$ & $0.27(0.13,0.41)$ & $0.0002 * \dagger$ & $0.28(0.13,0.43)$ & $0.0003 * \dagger$ \\
\hline Leptin (nmol/1) & $4.54(1.96,7.11)$ & $0.0006 * \dagger$ & $4.57(2.02,7.11)$ & $0.0005 * \dagger$ & $4.73(2.15,7.31)$ & $0.0004 * \dagger$ \\
\hline Adiponectin $(\mu \mathrm{mol} / \mathrm{l})$ & $-4.00(-6.76,-1.23)$ & $0.005^{*}$ & $-4.03(-6.80,-1.26)$ & $0.005^{*}$ & $-3.80(-6.64,-0.96)$ & $0.009 *$ \\
\hline
\end{tabular}

Data are shown as $\beta(95 \% \mathrm{CI})$ for Factor 4 at $\mathrm{T} 1$ with health outcomes from $\mathrm{T} 1$ to $\mathrm{T} 2$

Model 1: adjusted for age and race/ethnicity

Model 2: Model $1+$ pubertal status (based on pubic hair development in boys and breast development in girls)

Model 3: Model $2+$ physical activity (mean METs/day over a 3-day period) + total energy intake (kJ/day)

${ }^{a}$ Age- and sex-specific $z$ score according to the WHO Growth Reference for children aged 5-19 years [37]

${ }^{\mathrm{b}}$ Sum of the subscapular, suprailiac and tricep skinfold thicknesses

* Significant at $\alpha=0.05 ;{ }^{\dagger}$ Significant after Bonferroni correction (boys and girls were analysed separately within each model; $\alpha<0.05 / 20=0.003$ )

carnitine, even after adjustment for maternal BMI at the time of OGTT. Additionally, lower maternal insulin sensitivity was differentially associated with cord blood levels of branched chain amino acids and acylcarnitines. In another study of mother-infant pairs $(n=119$ from the PREOBE cohort in Spain), Shokry et al [19] leveraged targeted metabolomics data and identified associations of maternal GDM with elevated levels of cord blood hexoses, and lower levels of free carnitines, acylcarnitines, long-chain non-esterified fatty acids, phospholipids, Kreb's cycle metabolites and $\beta$-oxidation markers. While the metabolites in Factor 4 do not markedly overlap with those identified in either of the above-mentioned studies (with the exception of the relevance of compounds on phospholipid pathways to GDM in Shokry et al [19]), it is difficult to reconcile findings from untargeted platforms, which capture relative concentrations of all detectable metabolites in a given biotissue or fluid, with those of targeted platforms selected based on an investigator's a priori hypothesis of specific biological pathways. Further, it may not be appropriate to compare metabolites in fasting serum of youth with 
that of metabolites in cord blood, the latter of which are affected by a multitude of factors surrounding circumstances of delivery, and variability in metabolite concentrations across the maternal/fetal unit.

Associations of GDM with metabolites throughout late childhood and adolescence GDM exposure was associated with key metabolites within Factor 4 at a similar direction, magnitude, and precision of estimates at $\mathrm{T} 1$ and $\mathrm{T} 2$, indicating a persistent and consistent inter-individual effect of GDM on metabolite profiles from late childhood through adolescence. On the other hand, within-individual stability of the metabolites across $\mathrm{T} 1$ and $\mathrm{T} 2$ was modest at best, as indicated by relatively low ICCs. Our results suggest that, while the metabolites we examined may be informative for the study of disease aetiology given that the inter-individual rank of exposure/metabolite associations was preserved over time, concentrations of the specific metabolites are not likely to be reliable biomarkers of disease risk in youth within the age range of this study population due to high intra-individual variability.

Associations of metabolite patterns with adiposity and conventional metabolic risk biomarkers We found that a higher score for Factor 4 corresponded with greater adiposity, reduced insulin sensitivity, higher leptin and marginally lower adiponectin from late childhood through adolescence among girls. Furthermore, all associations except for that of GDM with adiponectin passed Bonferroni correction.

In a recent study in non-human primates, Polewski et al identified differences in plasma phospholipid composition, most notably perturbations in diacylglycerols that are desaturation products of palmitic acid, as markers of worsening insulin resistance and onset of the metabolic syndrome [56]. While the specific metabolites identified differed from those of the present study, phospholipids are integral components of the cell membrane and plasma and their metabolism influences numerous biochemical pathways, including those involved in glycaemic regulation [57]. The compounds of particular interest in Factor 4 include diacylglycerol derivatives of palmitoleic acid (e.g., palmitoleoyl-linoleoyl-glycerol [16:1/18:2]), an unsaturated monounsaturated fatty acid that has been found to exhibit similar detrimental effects to palmitic acid on cardiovascular traits in adult men [58]. Although, this lipokine has also been linked to beneficial effects on other aspects of metabolism (e.g., amelioration or prevention of insulin resistance and diabetes) [59].

Given the established literature on the puberty-related increase in adiposity [60] and insulin resistance [61], the female-specific nature of our findings is likely to be related to an association of maternal GDM with earlier adrenarche and pubarche in daughters. Recent cohort studies, including one in EPOCH [62], demonstrated that in utero exposure to
GDM was associated with earlier puberty onset, with stronger [62] or sole effects among female offspring [63-66]. This phenomenon may operate through a programming effect of maternal hyperglycaemia on offspring adrenal hormone production [67]. Future studies with longer term follow-up are necessary to establish whether the relationships of GDM exposure with adiposity and metabolic risk in the offspring persists beyond puberty. Additionally, studies should further investigate potential sex-specific programming effects of in utero GDM exposure on offspring adiposity and metabolic health.

\section{Strengths and weaknesses}

Our study has several strengths. First, we were able to examine long-term effects of intrauterine exposure to maternal GDM on fasting serum metabolites at, not just one, but two time points, from late childhood through adolescence. Our repeated metabolomics measurements enabled us to assess both inter-individual preservation of the effect of GDM exposure over time, as well as intra-individual variability of metabolites, of which the former is informative for understanding disease aetiology, while the latter has important implications for use of metabolites as prognostic biomarkers [9]. Second, we had a large sample size, especially in comparison with other studies of metabolomics in youth, where $N$ is less than 300 [50, 68-72]. Third, we were able to use rich data on sociodemographic and lifestyle characteristics to account for variables that may confound associations of interest. Finally, we had a diverse study sample, with $\sim 50 \%$ of participants of racial or ethnic minorities.

This study also has several limitations. First, we are not able to make inference on metabolic flux given that the repeated metabolic assessments were separated by a 5 -year period. Second, the metabolite factor scores were based on metabolites assayed at the same time as the first measurement of adiposity and metabolic biomarkers and, thus, estimates could be driven by cross-sectional relationships rather than being true prospective associations. Third, missing values and recall errors in self-reported covariates can introduce bias into estimates of association. Fourth, removal of metabolites with high missingness $(\geq 20 \%)$ from our dataset resulted in retention of $\sim 64 \%$ of the original features detected by the untargeted platform. Since unsupervised dimension reduction techniques, like PCA, rely on the intercorrelations among variables in the dataset, removal of features is likely to have had an impact on the composition of the factor scores in ways that we are not able to directly assess given that many of these procedures (including PCA) also require that there are no missing values in the input dataset. Fifth, GDM status was based on a clinical diagnosis of GDM following an OGTT, for which we do not have access to actual blood glucose concentrations. Thus, clinical misdiagnoses of GDM status could introduce non- 
differential bias into estimates of association pertaining to the relationship between maternal GDM and the metabolite factor scores. Sixth, while EPOCH participants were asked to arrive for the research visit having fasted for at least $8 \mathrm{~h}$, we do not know for certain how long they had truly been fasting; this is another source of non-differential bias, but this time with respect to the associations of factor scores with adiposity and metabolic risk from childhood through adolescence. Seventh, we cannot discount the possibility of false-positive findings given the large number of models tested, especially given that our initial assessment of the relationship between GDM exposure and offspring metabolite patterns was largely null (with the exception of the association of GDM with Factor 4 when dichotomised as Q4 vs Q1-3 using a nominal $\alpha=0.05$ ). However, we put forth precautionary measures to prevent type 1 error, including: (1) use of PCA as a data reduction method to identify metabolite factors in offspring that differed with respect to maternal GDM using a nominal $p$ value cut-off before assessing associations of GDM with individual metabolites; and (2) employment of more stringent $p$ value cut-offs in analyses of associations between GDM and metabolites, and between the metabolite pattern factor score and health outcomes. Finally, as with all cohort studies that span a long period of time between assessment of the exposure and measurement of the health outcome, there is potential for residual confounding due to unmeasured lifestyle characteristics (e.g., nutritional factors, more nuanced assessments of physical activity and energy expenditure), and shared behavioural and environmental characteristics between the mother and child.

\section{Conclusions and future directions}

In the EPOCH cohort, we identified a phospholipid metabolite pattern following intrauterine exposure to maternal GDM that was detectable from late childhood through adolescence. This metabolite pattern was associated with higher adiposity, worse insulin sensitivity and altered adipocytokines across the adolescent transition among girls exposed to in utero GDM vs those unexposed. Future research is required to evaluate the extent to which these metabolite patterns are clinically relevant and meaningfully associated with health outcomes beyond the adolescent transition.

Acknowledgements We thank the EPOCH participants, as well as past and present research assistants.

Data availability Data are available upon request.

Funding This study was supported by the National Institutes of Health $(\mathrm{NIH})$, National Institute of Diabetes, Digestive, and Kidney Diseases (R01 DK068001). The funders had no role in the design, conduct or reporting of this work.
Duality of interest GM is an employee at Metabolon, Inc. All other authors declare that there is no duality of interest associated with this manuscript.

Contribution statement WP and DD conceived the research question. WP is the guarantor of this work. WP conducted the analysis, wrote the initial draft of the paper and incorporated co-author comments. BMR, KMK and HAS curated the data and provided feedback on the analysis. GM oversaw the laboratory analysis for the metabolomics assays and participated in the writing of the manuscript. BMR, HAS, KMK and DD revised the article critically for important intellectual content. All co-authors approved the final version of the paper.

\section{References}

1. Kim SY, England JL, Sharma JA, Njoroge T (2011) Gestational diabetes mellitus and risk of childhood overweight and obesity in offspring: a systematic review. Exp Diabetes Res 2011:541308. https://doi.org/10.1155/2011/541308

2. Catalano PM, Thomas A, Huston-Presley L, Amini SB (2003) Increased fetal adiposity: a very sensitive marker of abnormal in utero development. Am J Obstet Gynecol 189(6):1698-1704. https://doi.org/10.1016/s0002-9378(03)00828-7

3. Sauder KA, Hockett CW, Ringham BM, Glueck DH, Dabelea D (2017) Fetal overnutrition and offspring insulin resistance and betacell function: the Exploring Perinatal Outcomes among Children (EPOCH) study. Diabet Med 34(10):1392-1399. https://doi.org/ 10.1111/dme.13417

4. Krishnaveni GV, Veena SR, Hill JC, Kehoe S, Karat SC, Fall CH (2010) Intrauterine exposure to maternal diabetes is associated with higher adiposity and insulin resistance and clustering of cardiovascular risk markers in Indian children. Diabetes Care 33(2):402-404. https://doi.org/10.2337/dc09-1393

5. Gautier JF, Wilson C, Weyer C et al (2001) Low acute insulin secretory responses in adult offspring of people with early onset type 2 diabetes. Diabetes 50(8):1828-1833

6. West NA, Crume TL, Maligie MA, Dabelea D (2011) Cardiovascular risk factors in children exposed to maternal diabetes in utero. Diabetologia 54(3):504-507. https://doi.org/10.1007/ s00125-010-2008-1

7. Wright CS, Rifas-Shiman SL, Rich-Edwards JW, Taveras EM, Gillman MW, Oken E (2009) Intrauterine exposure to gestational diabetes, child adiposity, and blood pressure. Am J Hypertens 22(2):215-220. https://doi.org/10.1038/ajh.2008.326

8. Bunt JC, Tataranni PA, Salbe AD (2005) Intrauterine exposure to diabetes is a determinant of hemoglobin $\mathrm{A}_{1} \mathrm{c}$ and systolic blood pressure in pima Indian children. J Clin Endocrinol Metab 90(6): 3225-3229. https://doi.org/10.1210/jc.2005-0007

9. Hivert MF, Perng W, Watkins SM et al (2015) Metabolomics in the developmental origins of obesity and its cardiometabolic consequences. J Dev Orig Health Dis 6(2):65-78. https://doi.org/10. 1017/s204017441500001x

10. Bentley-Lewis R, Huynh J, Xiong G et al (2015) Metabolomic profiling in the prediction of gestational diabetes mellitus. Diabetologia 58(6):1329-1332. https://doi.org/10.1007/s00125015-3553-4

11. de Seymour JV, Conlon CA, Sulek K et al (2014) Early pregnancy metabolite profiling discovers a potential biomarker for the subsequent development of gestational diabetes mellitus. Acta Diabetol 51(5):887-890. https://doi.org/10.1007/s00592-014-0626-7

12. Diaz SO, Pinto J, Graca G et al (2011) Metabolic biomarkers of prenatal disorders: an exploratory NMR metabonomics study of 
second trimester maternal urine and blood plasma. J Proteome Res 10(8):3732-3742. https://doi.org/10.1021/pr200352m

13. Enquobahrie DA, Denis M, Tadesse MG, Gelaye B, Ressom HW, Williams MA (2015) Maternal early pregnancy serum metabolites and risk of gestational diabetes mellitus. J Clin Endocrinol Metab 100(11):4348-4356. https://doi.org/10.1210/jc.2015-2862

14. Graca G, Duarte IF, Barros AS et al (2010) Impact of prenatal disorders on the metabolic profile of second trimester amniotic fluid: a nuclear magnetic resonance metabonomic study. J Proteome Res 9(11):6016-6024. https://doi.org/10.1021/ pr100815q

15. Nevalainen J, Sairanen M, Appelblom H, Gissler M, Timonen S, Ryynanen M (2016) First-trimester maternal serum amino acids and acylcarnitines are significant predictors of gestational diabetes. Rev Diabet Stud 13(4):236-245. https://doi.org/10.1900/rds.2016.13. 236

16. Pinto J, Almeida LM, Martins AS et al (2015) Prediction of gestational diabetes through NMR metabolomics of maternal blood. $\mathrm{J}$ Proteome Res 14(6):2696-2706. https://doi.org/10.1021/acs. jproteome.5b00260

17. Pinto J, Barros AS, Domingues MR et al (2015) Following healthy pregnancy by NMR metabolomics of plasma and correlation to urine. J Proteome Res 14(2):1263-1274. https://doi.org/10.1021/ pr5011982

18. Lowe WL Jr, Bain JR, Nodzenski M et al (2017) Maternal BMI and glycemia impact the fetal metabolome. Diabetes Care 40(7):902910. https://doi.org/10.2337/dc16-2452

19. Shokry E, Marchioro L, Uhl O et al (2019) Impact of maternal BMI and gestational diabetes mellitus on maternal and cord blood metabolome: results from the PREOBE cohort study. Acta Diabetol 56(4):421-430. https://doi.org/10.1007/s00592-019-01291-z

20. Spalding KL, Arner E, Westermark PO et al (2008) Dynamics of fat cell turnover in humans. Nature 453(7196):783-787. https://doi. org/10.1038/nature06902

21. Alberga AS, Sigal RJ, Goldfield G, Prud'homme D, Kenny GP (2012) Overweight and obese teenagers: why is adolescence a critical period? Pediatric Obesity 7(4):261-273. https://doi.org/10. 1111/j.2047-6310.2011.00046.x

22. Wang TJ, Larson MG, Vasan RS et al (2011) Metabolite profiles and the risk of developing diabetes. Nat Med 17(4):448-453. https://doi.org/10.1038/nm.2307

23. Crume TL, Ogden L, West NA et al (2011) Association of exposure to diabetes in utero with adiposity and fat distribution in a multiethnic population of youth: the Exploring Perinatal Outcomes among Children (EPOCH) study. Diabetologia 54(1):87-92. https://doi. org/10.1007/s00125-010-1925-3

24. National Diabetes Data Group (1979) Classification and diagnosis of diabetes mellitus and other categories of glucose intolerance. Diabetes 28(12):1039-1057. https://doi.org/10.2337/diab.28.12. 1039

25. Crume TL, Ogden L, Daniels S, Hamman RF, Norris JM, Dabelea D (2011) The impact of in utero exposure to diabetes on childhood body mass index growth trajectories: the EPOCH study. J Pediatr 158(6):941-946. https://doi.org/10.1016/j.jpeds.2010.12.007

26. Crume TL, Ogden L, Maligie M et al (2011) Long-term impact of neonatal breastfeeding on childhood adiposity and fat distribution among children exposed to diabetes in utero. Diabetes Care 34(3): 641-645. https://doi.org/10.2337/dc10-1716

27. Smilde AK, van der Werf MJ, Bijlsma S, van der Werff-van der Vat BJC, Jellema RH (2005) Fusion of mass spectrometry-based metabolomics data. Anal Chem 77(20):6729-6736. https://doi.org/10. 1021/ac051080y

28. Schiffman C, Petrick L, Perttula K et al (2019) Filtering procedures for untargeted LC-MS metabolomics data. BMC Bioinformatics 20(1):334. https://doi.org/10.1186/s12859-019-2871-9
29. Hastie T, Tibshirani R, Sherlock G, Eisen M, Brown P, Botstein D (1999) Imputing missing data for gene expression arrays. Available from www.web.stanford.edu/ hastie/Papers/missing.pdf. Accessed 10 October 2019

30. Risso D, Ngai J, Speed TP, Dudoit S (2014) Normalization of RNA-seq data using factor analysis of control genes or samples. Nat Biotechnol 32(9):896-902. https://doi.org/10.1038/nbt.2931

31. Conwell LS, Trost SG, Brown WJ, Batch JA (2004) Indexes of insulin resistance and secretion in obese children and adolescents: a validation study. Diabetes Care 27(2):314-319. https://doi.org/10. 2337/diacare.27.2.314

32. George L, Bacha F, Lee S, Tfayli H, Andreatta E, Arslanian S (2011) Surrogate estimates of insulin sensitivity in obese youth along the spectrum of glucose tolerance from normal to prediabetes to diabetes. J Clin Endocrinol Metab 96(7):2136-2145. https://doi. org/10.1210/jc. $2010-2813$

33. Gungor N, Saad R, Janosky J, Arslanian S (2004) Validation of surrogate estimates of insulin sensitivity and insulin secretion in children and adolescents. J Pediatr 144(1):47-55. https://doi.org/ 10.1016/j.jpeds.2003.09.045

34. Yajnik CS, Katre PA, Joshi SM et al (2015) Higher glucose, insulin and insulin resistance (HOMA-IR) in childhood predict adverse cardiovascular risk in early adulthood: the Pune Children's Study. Diabetologia 58(7):1626-1636. https://doi.org/10.1007/s00125015-3602-Z

35. Cancello R, Tounian A, Poitou C, Clement K (2004) Adiposity signals, genetic and body weight regulation in humans. Diabetes Metab 30(3):215-227

36. Fleisch AF, Agarwal N, Roberts MD et al (2007) Influence of serum leptin on weight and body fat growth in children at high risk for adult obesity. J Clin Endocrinol Metab 92(3):948-954. https://doi. org/10.1210/jc.2006-1390

37. Freedman DS, Sherry B (2009) The validity of BMI as an indicator of body fatness and risk among children. Pediatrics 124(Suppl 1): S23-S34. https://doi.org/10.1542/peds.2008-3586E

38. Boeke CE, Oken E, Kleinman KP, Rifas-Shiman SL, Taveras EM, Gillman MW (2013) Correlations among adiposity measures in school-aged children. BMC Pediatr 13:99. https://doi.org/10.1186/ 1471-2431-13-99

39. Wells JCK, Fewtrell MS (2006) Measuring body composition. Arch Dis Child 91(7):612-617. https://doi.org/10.1136/adc.2005. 085522

40. Centers for Disease Control and Prevention (2007) National Health and Nutrition Examination Survey (NHANES). Available from wwwn.cdc.gov/nchs/nhanes/ContinuousNhanes/Default.aspx? BeginYear=2007. Accessed 10 October 2019

41. Marshall WA, Tanner JM (1968) Growth and physiological development during adolescence. Annu Rev Med 19:283-300. https:// doi.org/10.1146/annurev.me.19.020168.001435

42. Chavarro JE, Watkins DJ, Afeiche MC et al (2017) Validity of selfassessed sexual maturation against physician assessments and hormone levels. J Pediatr 186:172-178.e3. https://doi.org/10. 1016/j.jpeds.2017.03.050

43. Weston AT, Petosa R, Pate RR (1997) Validation of an instrument for measurement of physical activity in youth. Med Sci Sports Exerc 29(1):138-143. https://doi.org/10.1097/00005768199701000-00020

44. Cullen KW, Watson K, Zakeri I (2008) Relative reliability and validity of the Block Kids Questionnaire among youth aged 10 to 17 years. J Am Diet Assoc 108(5):862-866. https://doi.org/10. 1016/j.jada.2008.02.015

45. Perng W, Gillman MW, Mantzoros CS, Oken E (2014) A prospective study of maternal prenatal weight and offspring cardiometabolic health in midchildhood. Ann Epidemiol 24(11):793-800.e1. https://doi.org/10.1016/j.annepidem.2014.08.002 
46. Shapiro AL, Schmiege SJ, Brinton JT et al (2015) Testing the fuelmediated hypothesis: maternal insulin resistance and glucose mediate the association between maternal and neonatal adiposity, the Healthy Start study. Diabetologia 58(5):937-941. https://doi.org/ 10.1007/s00125-015-3505-z

47. Perng W, Mora-Plazas M, Marin C, Rozek LS, Baylin A, Villamor E (2013) A prospective study of LINE-1DNA methylation and development of adiposity in school-age children. PLoS One 8(4): e62587. https://doi.org/10.1371/journal.pone.0062587

48. Breton CV, Marsit CJ, Faustman E et al (2017) Small-magnitude effect sizes in epigenetic end points are important in children's environmental health studies: the Children's Environmental Health and Disease Prevention Research Center's Epigenetics Working Group. Environ Health Perspect 125(4):511-526. https:// doi.org/10.1289/ehp595

49. Perng WOE, Dabelea D (2019) Developmental overnutrition and obesity and type 2 diabetes in offspring. Diabetologia. 62(10): 1779-1788. https://doi.org/10.1007/s00125-019-4914-1

50. Perng W, Gillman MW, Fleisch AF et al (2014) Metabolomic profiles and childhood obesity. Obesity 22(12):2570-2578. https://doi.org/10.1002/oby.20901

51. Berglund SK, Garcia-Valdes L, Torres-Espinola FJ et al (2016) Maternal, fetal and perinatal alterations associated with obesity, overweight and gestational diabetes: an observational cohort study (PREOBE). BMC Public Health 16:207. https://doi.org/10.1186/ s12889-016-2809-3

52. National Center for Biotechnology Information. PubChem Database. CID=9543695. Available from https://pubchem.ncbi. nlm.nih.gov/compound/1-Palmitoyl-2-linoleoyl-sn-glycerol. Accessed 10 October 2019

53. Paton CM, Ntambi JM (2009) Biochemical and physiological function of stearoyl-CoA desaturase. Am J Physiol Endocrinol Metab 297(1):E28-E37. https://doi.org/10.1152/ajpendo.90897.2008

54. Dobrzyn P, Jazurek M, Dobrzyn A (2010) Stearoyl-CoA desaturase and insulin signaling - what is the molecular switch? Biochim Biophys Acta 1797(6-7):1189-1194. https://doi.org/10.1016/j. bbabio.2010.02.007

55. Rahman SM, Dobrzyn A, Lee SH, Dobrzyn P, Miyazaki M, Ntambi JM (2005) Stearoyl-CoA desaturase 1 deficiency increases insulin signaling and glycogen accumulation in brown adipose tissue. Am J Physiol Endocrinol Metab 288(2):E381-E387. https://doi.org/10. 1152/ajpendo.00314.2004

56. Polewski MA, Burhans MS, Zhao M et al (2015) Plasma diacylglycerol composition is a biomarker of metabolic syndrome onset in rhesus monkeys. J Lipid Res 56(8):1461-1470. https://doi.org/10. 1194/jlr.M057562

57. Chang W, Hatch GM, Wang Y, Yu F, Wang M (2019) The relationship between phospholipids and insulin resistance: from clinical to experimental studies. J Cell Mol Med 23(2):702-710. https://doi. org/10.1111/jcmm.13984

58. Nestel P, Clifton P, Noakes M (1994) Effects of increasing dietary palmitoleic acid compared with palmitic and oleic acids on plasma lipids of hypercholesterolemic men. J Lipid Res 35(4):656-662

59. Frigolet ME, Gutierrez-Aguilar R (2017) The role of the novel lipokine palmitoleic acid in health and disease. Adv Nutr 8(1): 173s-181s. https://doi.org/10.3945/an.115.011130
60. Siervogel RM, Demerath EW, Schubert C et al (2003) Puberty and body composition. Horm Res 60(Suppl 1):36-45. https://doi.org/ 10.1159/000071224

61. Kelsey MM, Zeitler PS (2016) Insulin resistance of puberty. Curr Diab Rep 16(7):64. https://doi.org/10.1007/s11892-016-0751-5

62. Hockett CW, Bedrick EJ, Zeitler P, Crume TL, Daniels S, Dabelea D (2019) Exposure to diabetes in utero is associated with earlier pubertal timing and faster pubertal growth in the offspring: the EPOCH study. J Pediatr 206:105-112. https://doi.org/10.1016/j. jpeds.2018.10.053

63. Kubo A, Ferrara A, Laurent CA et al (2016) Associations between maternal pregravid obesity and gestational diabetes and the timing of pubarche in daughters. Am J Epidemiol 184(1):7-14. https://doi. org/10.1093/aje/kww006

64. Lauridsen LLB, Arendt LH, Ernst A et al (2018) Maternal diabetes mellitus and timing of pubertal development in daughters and sons: a nationwide cohort study. Fertil Steril 110(1):35-44. https://doi. org/10.1016/j.fertnstert.2018.03.014

65. Lawn RB, Lawlor DA, Fraser A (2018) Associations between maternal prepregnancy body mass index and gestational weight gain and daughter's age at menarche: the Avon Longitudinal Study of Parents and Children. Am J Epidemiol 187(4):677-686. https://doi.org/10.1093/aje/kwx308

66. Grunnet LG, Hansen S, Hjort L et al (2017) Adiposity, dysmetabolic traits, and earlier onset of female puberty in adolescent offspring of women with gestational diabetes mellitus: a clinical study within the Danish National Birth Cohort. Diabetes Care 40(12):1746-1755. https://doi.org/10.2337/dc17-0514

67. Poston L (2010) Developmental programming and diabetes - the human experience and insight from animal models. Best Pract Res Clin Endocrinol Metab 24(4):541-552. https://doi.org/10.1016/j. beem.2010.05.007

68. Michaliszyn SF, Sjaarda LA, Mihalik SJ et al (2012) Metabolomic profiling of amino acids and beta-cell function relative to insulin sensitivity in youth. J Clin Endocrinol Metab 97(11):E2119-E2124. https://doi.org/10.1210/jc.2012-2170

69. Perng W, Oken E, Roumeliotaki T et al (2016) Leptin, acylcarnitine metabolites and development of adiposity in the Rhea mother-child cohort in Crete, Greece. Obes Sci Pract 2(4):471-476. https://doi. org/10.1002/osp4.65

70. Perng W, Hector EC, Song PXK et al (2017) Metabolomic determinants of metabolic risk in Mexican adolescents. Obesity 25(9): 1594-1602. https://doi.org/10.1002/oby.21926

71. Mihalik SJ, Michaliszyn SF, de las Heras J et al (2012) Metabolomic profiling of fatty acid and amino acid metabolism in youth with obesity and type 2 diabetes: evidence for enhanced mitochondrial oxidation. Diabetes Care 35(3):605-611. https://doi. org $10.2337 / \mathrm{dc} 11-1577$

72. Butte NF, Liu Y, Zakeri IF et al (2015) Global metabolomic profiling targeting childhood obesity in the Hispanic population. Am J Clin Nutr 102(2):256-267. https://doi.org/10.3945/ajen.115. 111872

Publisher's note Springer Nature remains neutral with regard to jurisdictional claims in published maps and institutional affiliations. 